Portland State University

PDXScholar

3-1-2006

\title{
Nonlocal effects on optical and molecular interactions with metallic nanoshells
}

\author{
P.T. Leung \\ Portland State University \\ Railing Chang \\ National Taiwan Ocean University
}

Follow this and additional works at: https://pdxscholar.library.pdx.edu/phy_fac

Part of the Physics Commons

Let us know how access to this document benefits you.

\section{Citation Details}

Railing, C., \& Leung, P. T. (2006). Nonlocal effects on optical and molecular interactions with metallic nanoshells. Physical Review B (Condensed Matter And Materials Physics), 73(12), 125438-1-6.

This Article is brought to you for free and open access. It has been accepted for inclusion in Physics Faculty Publications and Presentations by an authorized administrator of PDXScholar. Please contact us if we can make this document more accessible: pdxscholar@pdx.edu. 


\title{
Nonlocal effects on optical and molecular interactions with metallic nanoshells
}

\author{
Railing Chang ${ }^{1}$ and P. T. Leung ${ }^{2}$ \\ ${ }^{1}$ Institute of Optoelectronic Sciences, National Taiwan Ocean University, Keelung, Taiwan, Republic of China \\ and Center of Nanostorage Research, National Taiwan University, Taipei 10617, Taiwan, Republic of China \\ ${ }^{2}$ Department of Physics, Portland State University, P. O. Box 751, Portland, Oregon 97207-0751, USA
}

(Received 29 April 2005; revised manuscript received 23 January 2006; published 28 March 2006)

\begin{abstract}
Theoretical studies of the optical response of metallic nanoshells have been carried out, where quantum effects are partially accounted for through the application of a nonlocal response model for shells of mesoscopic dimensions. Both far field and near field interactions are considered, with the incident source being a plane wave and an emitting molecular dipole, respectively. It is found that these nonlocal effects can lead to significant deviations from macroscopic electrodynamic theory, for shells of ultrasmall dimensions $(<10 \mathrm{~nm})$ or ultrathin thickness $(\sim 1 \mathrm{~nm})$, and are particularly significant for processes involving higher multipolar responses of the nanoshells. It is further concluded that these effects can still be observable, even in the presence of possibly large interfacial scattering for the free electrons in these nanoshells.
\end{abstract}

DOI: 10.1103/PhysRevB.73.125438 PACS number(s): 78.67.Bf, 73.22.Lp, 78.40.Kc, 41.20.Cv

\section{INTRODUCTION}

Since its first successful synthesis using chemical methods in the late $1990 \mathrm{~s},{ }^{1}$ the metallic nanoshell has become a unique plasmonic system and has been very actively studied in the past decade. ${ }^{1-11}$ This is due both to the capability of these shells in enhancing various optical processes, as well as the high tunability in their plasmon resonance frequencies via the control of the shell thickness relative to the size of the core. Experimentally, both dielectric ${ }^{1-3}$ and hollow ${ }^{4,5}$ cores can be fabricated using methods of colloidal chemistry. Furthermore, by varying the shell thickness towards the thin shell limit (in the order of a few $\mathrm{nm}$ ), the plasmon frequency can be adjusted across the whole visible spectrum-from near UV to near IR. ${ }^{2}$

Theoretically, most of the previous investigations on the optical property of this system have applied classical electrodynamics in the form of the Mie scattering theory, ${ }^{2,3,10-12}$ although in a few cases microscopic theory such as the timedependent density functional theory (TDDFT) has also been formulated. ${ }^{6,7}$ While the Mie approach is limited to the bulk dielectric response of the nanoshell, the previous application of TDDFT is mostly limited to dipolar response of the system, as in the study of the photoabsorption by these shells. ${ }^{6,7}$ While dipolar response is sometimes sufficient to account for light scattering and absorption phenomena from these nanostructures, ${ }^{13}$ higher mulipolar response is necessary in describing the interaction between emitting molecular species in the vicinity of these structures, such as in problems involving enhanced molecular fluorescence and surfaceenhanced Raman scattering (SERS). ${ }^{2}$ Moreover, the TDDFT approach, even when it is extended to treat multipolar response, is often limited to very small sizes $(\sim 1-2 \mathrm{~nm})$ of the structure. ${ }^{14}$ It will be therefore of interest if one can investigate the optical response of these nanoshells to all order of multipoles, accounting for the microscopic quantum mechanical nature of the metallic electrons in these systems of mesoscopic sizes (e.g., $\sim 10 \mathrm{~nm}$ ).

It is the purpose of the present work to apply a nonlocal (NL) model previously established in the literature to study the optical response of these ultrasmall metallic nanoshells, accounting for quantum effects through the application of appropriate NL dielectric functions. ${ }^{15}$ We shall also account for the scattering between the metallic electrons and the shell boundaries. This can lead to a damping rate in the dielectric function much greater than the one for bulk metals and can possibly suppress the NL effects. We shall see below that despite this damping, the NL effects are still likely observable in processes involving multipolar interaction with the nanoshell.

\section{FORMULATION}

For structures of tens of nanometers which are much smaller than optical and UV wavelengths, quasistatic theory can be applied and their optical response can be determined largely by the multipole polarizabilities of the system. ${ }^{13}$ Accounting for NL response, we have recently ${ }^{15}$ studied such a theory for a spherical shell of inner radius $a$ and outer radius $b$, where we have assumed the region outside the shell $(b<r)$ to be vacuum and the inner core $(r<a)$ filled with a dielectric of real dielectric constant $\varepsilon^{\prime}$, while the shell region $(a<r<b)$ is filled with metal described by a NL isotropic dielectric function $\varepsilon(k, \omega)$ which is both temporal and spatial dispersive. For such a system, the NL polarizability has been derived previously in the literature via the semiclassical infinite barrier (SCIB) model. ${ }^{16}$ The main idea of this model is to assume a fictitious continuation of the metallic region beyond the geometrical boundaries at $r=a$ and $r=b$. By introducing an additional boundary condition $(\mathrm{ABC})$ to require the radial components of the displacement vectors $\left(D_{r}\right)$ to be discontinuous at the boundaries, which corresponds to the presence of a fictitious external surface charge at these boundaries, the electric field can hence be smoothly extended across the geometrical boundaries. In this SCIB model, the NL response of the metal can then be obtained using Fourier transform techniques. While the result described in Ref. 16 via the surface impedance concept is actually more general and can be applied to the case when both the core and shell 
are described by a NL dielectric function, we have reproduced their result for our nanoshell by directly matching the $\mathrm{ABC}$ across the boundaries. In slightly different notations, the $\ell$ th order NL polarizability can be expressed as follows: ${ }^{15,17}$

$$
\alpha_{\ell}=-\left(\frac{\ell \beta+b \gamma}{(\ell+1) \beta-b \gamma}\right) b^{2 \ell+1} \equiv \Delta_{\ell} b^{2 \ell+1},
$$

where the quantities $\beta$ and $\gamma$ are defined as follows:

$$
\begin{aligned}
& \beta= \ell \frac{a^{\ell+1}}{b^{\ell-1}} F(a, b)+(\ell+1) b^{2} F(b, b)+\frac{2}{\pi} \varepsilon^{\prime} a b^{2} \ell(2 \ell+1) \\
& \times[F(a, a) F(b, b)-F(a, b) F(b, a)], \\
& \gamma=\frac{\pi}{2} \frac{\ell(\ell+1)}{2 \ell+1}\left[\left(\frac{a}{b}\right)^{2 \ell+1}-1\right]-\varepsilon^{\prime} a \ell^{2} F(a, a) \\
&-\varepsilon^{\prime} \ell(\ell+1) \frac{a^{\ell+1}}{b^{\ell}} F(b, a),
\end{aligned}
$$

and the function $F(x, y)$ is defined in the form of an integral, ${ }^{16}$

$$
F(x, y)=\int_{0}^{\infty} \frac{j_{\ell}(k x) j_{\ell}(k y)}{\varepsilon(k, \omega)} d k .
$$

We have also derived explicitly the corresponding local limit of this model ${ }^{15}$ as shown in the Appendix. In the following, we shall present a numerical study of different aspects of the NL effects, including in particular the role of the shell thickness and the dielectric core on the NL response of these nanoshells. We shall illustrate the NL effects by comparing the results obtained from Eq. (1) with those from Eq. (A2) for the local case, and we shall study the interaction between both optical far fields and near fields with the nanoshell.

In the case with incident far fields, we shall study the extinction efficiency (absorption and scattering) for plane waves incident on these nanoshells. We shall limit to the dipole approximation (with light wavelength $\lambda \gg b \sim 10 \mathrm{~nm}$ ) in which the absorption and scattering efficiencies can be obtained from the following well-known expressions: ${ }^{9}$

$$
Q_{a b}=\frac{8 \pi}{\lambda} \operatorname{Im}\left(\Delta_{1}\right) b,
$$

and

$$
Q_{s c}=\frac{8}{3}\left(\frac{2 \pi b}{\lambda}\right)^{4}\left|\Delta_{1}\right|^{2} .
$$

In the case of near field interactions, we shall study the modified decay rate and emission frequency shift of an emitting molecule close to the shell. These can be obtained in a phenomenological model in terms of the natural decay rate as follows: ${ }^{18}$

$$
\Gamma_{M} / \Gamma_{0}=1+\frac{3 q}{2 k^{3}} \operatorname{Im}(G),
$$

$$
\Delta \omega_{M} / \Gamma_{0}=-\frac{3 q}{4 k^{3}} \operatorname{Re}(G),
$$

where $q, \Gamma_{0}$, and $k$ are respectively the quantum yield, natural decay rate, and the natural emission wave number of the molecule. In Eqs. (7) and (8), the $G$ factor is defined as

$$
G=\sum_{l} \Delta_{l} \frac{f_{l} b^{2 l+1}}{r^{2 l+4}},
$$

with $f_{l}=(l+1)^{2}\left[f_{l}=l(l+1) / 2\right]$ for tangential [radial] dipole and $r$ being the radial coordinate of the molecule. We shall study the NL effects on the results in Eqs. (5)-(8) for different size and thickness of the shell.

\section{NUMERICAL RESULTS AND DISCUSSION}

We have carried out some model calculations to illustrate the NL effects. For the dielectric response of the metal, we have adopted both the hydrodynamic and the LindhardMermin (LM) models. In the case of the hydrodynamic model, we have

$$
\varepsilon(k, \omega)=1-\frac{\omega_{p}^{2}}{\omega(\omega+i \Gamma)-v_{0}^{2} k^{2}},
$$

where $\omega_{p}$ and $\Gamma$ are the plasmon frequency and damping factor of the electrons, respectively, and $v_{0}=\sqrt{(3 / 5)} v_{F}$ with $v_{F}$ being the Fermi velocity; Eq. (4) can be integrated analytically to obtain

$$
\begin{aligned}
F(a, b)= & \frac{\pi}{2(2 \ell+1)}\left(\frac{a}{b}\right)^{\ell+1 / 2} \frac{1}{\sqrt{a b}} \\
& +\frac{\pi}{2 \sqrt{a b}}\left(\frac{\omega_{p}^{2}}{\omega_{p}^{2}-\omega(\omega+i \Gamma)}\right) I_{\ell+1 / 2}(\bar{\kappa} a) K_{\ell+1 / 2}(\bar{\kappa} b),
\end{aligned}
$$

where $I_{v}, K_{v}$ are modified Bessel functions, and $\bar{\kappa}$ $\equiv \frac{1}{v_{0}} \sqrt{\omega_{p}^{2}-\omega(\omega+i \Gamma)}$. In the case of the LM model, ${ }^{19}$ Eq. (4) can only be evaluated numerically. Note that we have ignored the interband term, for our focus here is in the NL effects on the free electron gas. This is in the same spirit as the approach in recent theoretical studies of radiative decay of plasmons in metallic nanoshells, ${ }^{12}$ with the understanding that the interband term can always be added to these dielectric functions if needed in the comparison with experiments. ${ }^{3}$

To numerically illustrate the NL effects, we shall limit our calculations to ultrasmall nanoshells of dimensions similar to those studied in Ref. 16. Although most of the previous experiments have studied nanoshells of greater dimensions, some studies have nevertheless reported nanoshells down to dimensions with a radius $\sim 10 \mathrm{~nm} .{ }^{9}$ Further experimental advance may soon come to even smaller dimensions, which will make our present calculations and the previous ones ${ }^{6,16}$ more relevant to observations. In the following, we shall study the NL effects for a silver nanoshell with the inside either hollow or filled with glass, the later being a common system studied in recent experimental works. ${ }^{8}$

Figure 1 shows the extinction for a silver shell of dimensions $a=2.5 \mathrm{~nm}$ and $b=3.0 \mathrm{~nm}$. As in previous work, ${ }^{16}$ bulk 

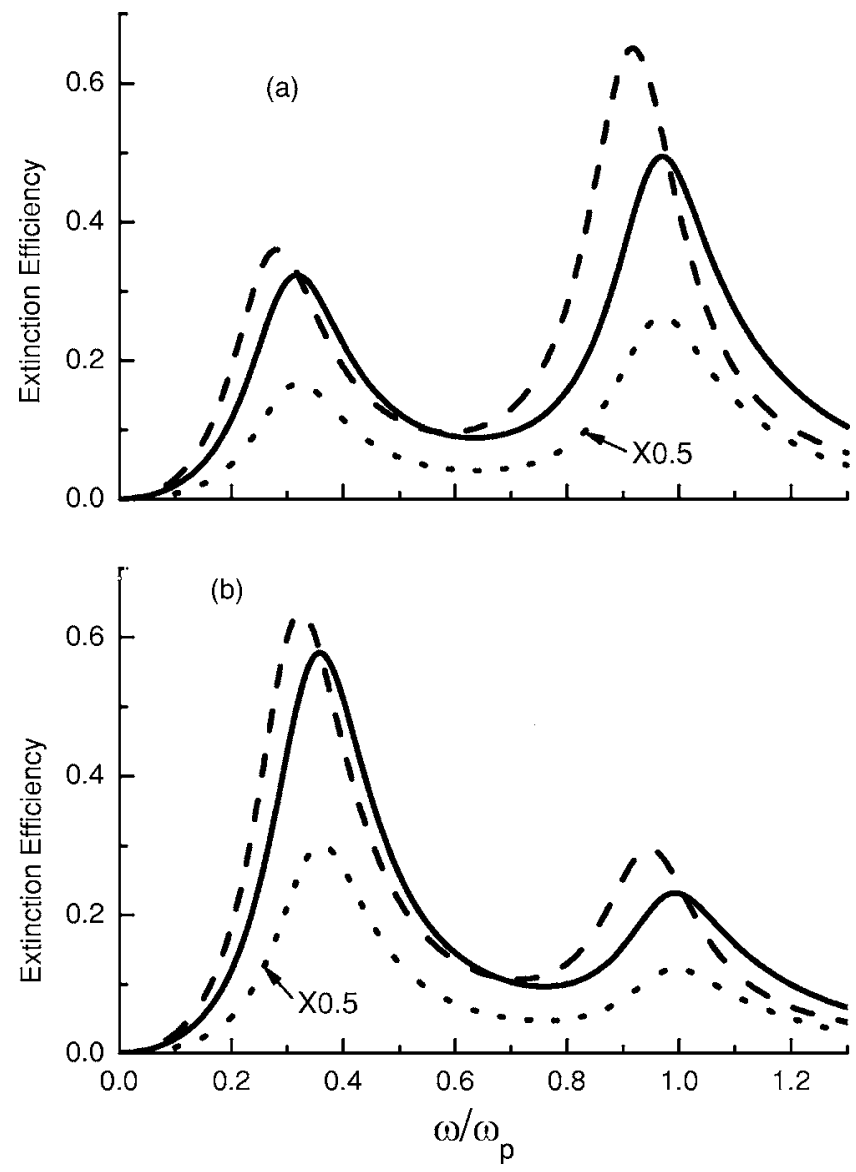

FIG. 1. Extinction efficiency of a silver nanoshell with inner radius $a=2.5 \mathrm{~nm}$ and outer radius $b=3.0 \mathrm{~nm}$ for (a) a glass core (b) a hollow core according to the Drude (dashed), hydrodynamic (dotted), and Lindhard-Mermin (solid) models, respectively. Note that the results for the hydrodynamic model are rescaled.

parameters for metal are used in our calculations. Thus, the plasmon frequency for $\mathrm{Ag}$ is taken as $\omega_{\mathrm{P}}=1.36 \times 10^{16} \mathrm{~s}^{-1}$, and the damping constant accounting for interfacial scattering has the following form: ${ }^{3,12} \Gamma=\Gamma_{\mathrm{BULK}}+A v_{F} /(b-a)$, with $\Gamma_{\mathrm{BULK}}=2.56 \times 10^{13} \mathrm{~s}^{-1}$ and $v_{F}=1.39 \times 10^{6} \mathrm{~m} / \mathrm{s}$ for silver. Without loss of generality, we have set the geometric factor $A=1$, in accord with recent simulation studies on these nanoshells. ${ }^{11}$ Previously, ${ }^{15}$ we have shown that the NL effects generally lead to a lower value of absorption and scattering for the nanoshell, with slightly blueshifted resonances ${ }^{16}$ in both the antisymmetric $\left(\omega_{+}\right)$and symmetric $\left(\omega_{-}\right)$coupled modes (see the Appendix for a discussion of these modes). This is also seen here for both the case of a Ag-glass nanoshell [ Fig. 1(a)] and a hollow Ag nanoshell [ Fig. 1(b)]. Also, it is found that both the hydrodynamic and LM models yield very close results (within a few \%) in the presence of such a relatively large surface-scattering damping effect $(A=1)$. Note that for clarity purpose, we have rescaled the hydrodynamic results in the figure. We have observed that the two models will differ in the prediction of additional "bulk resonances" 20 within the metal shell at frequencies above $\omega_{P}$ for smaller values of $A$.

By comparing the two results, it can be seen that the effect of filling the shell with glass is to redshift the reso- nance frequencies (both $\omega_{+}$and $\omega_{-}$), as observed previously in the literature. ${ }^{21}$ Furthermore, it is seen that the antisymmetric mode has a stronger extinction in the Ag-glass case, while the symmetric mode is weaker in extinction for this case. Usually, it is the symmetric mode which has a stronger absorption due to the overall larger induced dipole moment in this case. ${ }^{21}$ However, since these extinction coefficients are also proportional to the frequency, as is clear from Eqs. (5) and (6), the overall effect shows that when the shell is filled with glass, the absorption becomes even stronger for the $\omega_{+}$mode. On the other hand, in the hollow case, we reobtain a larger extinction for the $\omega_{-}$mode. We believe this is due to the fact that the $\omega_{-}$mode has a much greater absorption intensity than that of the $\omega_{+}$mode for the hollow case, as compared to the situation in the glass-filled case. ${ }^{21}$ Moreover, the blueshift due to the NL effects will lead to a smaller relative redshift between the Ag-glass and the hollow case for both the two plasmon modes.

Next we want to demonstrate that in the nanoshell case, the NL effects can be quite significant even for a relatively large shell with a very thin shell thickness. Figure 2 shows the real and imaginary parts of the spectral dipole polarizability for a Ag nanoshell of various metal thickness with an outer radius of $10 \mathrm{~nm}$. It is seen that when the shell thickness goes down from $5 \mathrm{~nm}$ to $1 \mathrm{~nm}$, the NL effects (only the LM model is shown) become relatively more prominent (with the peak shifts about twice as great). In particular, the magnitudes of the dispersion and the absorption are again suppressed (up to $\sim 12 \%$ ) by the NL effects in the case of very thin shells. Furthermore, the effects due to the increase in damping as the shell thickness decreases can also be clearly seen from the widths in the results of Fig. 2(b).

While the NL effects are relatively small in the above examples where we have limited to dipolar response for far field interaction with ultrasmall nanoshells, we next study the interaction of the nanoshell with a fluorescing molecular dipole in close proximity ${ }^{15}$ by calculating the quantities in Eqs. (7) and (8). As is clear from Eq. (9), many higher order multipolar responses will have to be included and we thus expect the NL effects to be more significant. ${ }^{16}$ This is understandable since higher multipolar response corresponds to excitations by radiation with large angular momentumimplying a large wave vector (momentum) along the nanoshell surfaces, which in turn implies larger significance of the NL effects. Figure 3 shows the spectrum of the normalized decay rate and the frequency shift for both a radial and a tangential molecular dipole fluorescing in the vicinity at $1 \mathrm{~nm}$ from a nanoshell. Although the results of both the hydrodynamic and Lindhard-Mermin models are again very close, one can observe their distinct feature compared to the Drude model (up to several times difference at resonance frequency). The shallow dip at $\omega=0.6 \omega_{p}\left(\omega=0.75 \omega_{p}\right)$ for the local (nonlocal) case corresponds to the gap between symmetric and antisymmetric modes. Since the calculation here involves the overall contributions from all higher order multipoles, the "peaks" in the spectrum are not as distinct as those in the simple dipole spectrum for the extinction coefficients. Rather, they will depend on the relative contribution of each of the multipole terms. Note that the frequency shift in the local case turns positive as the emission frequency of 

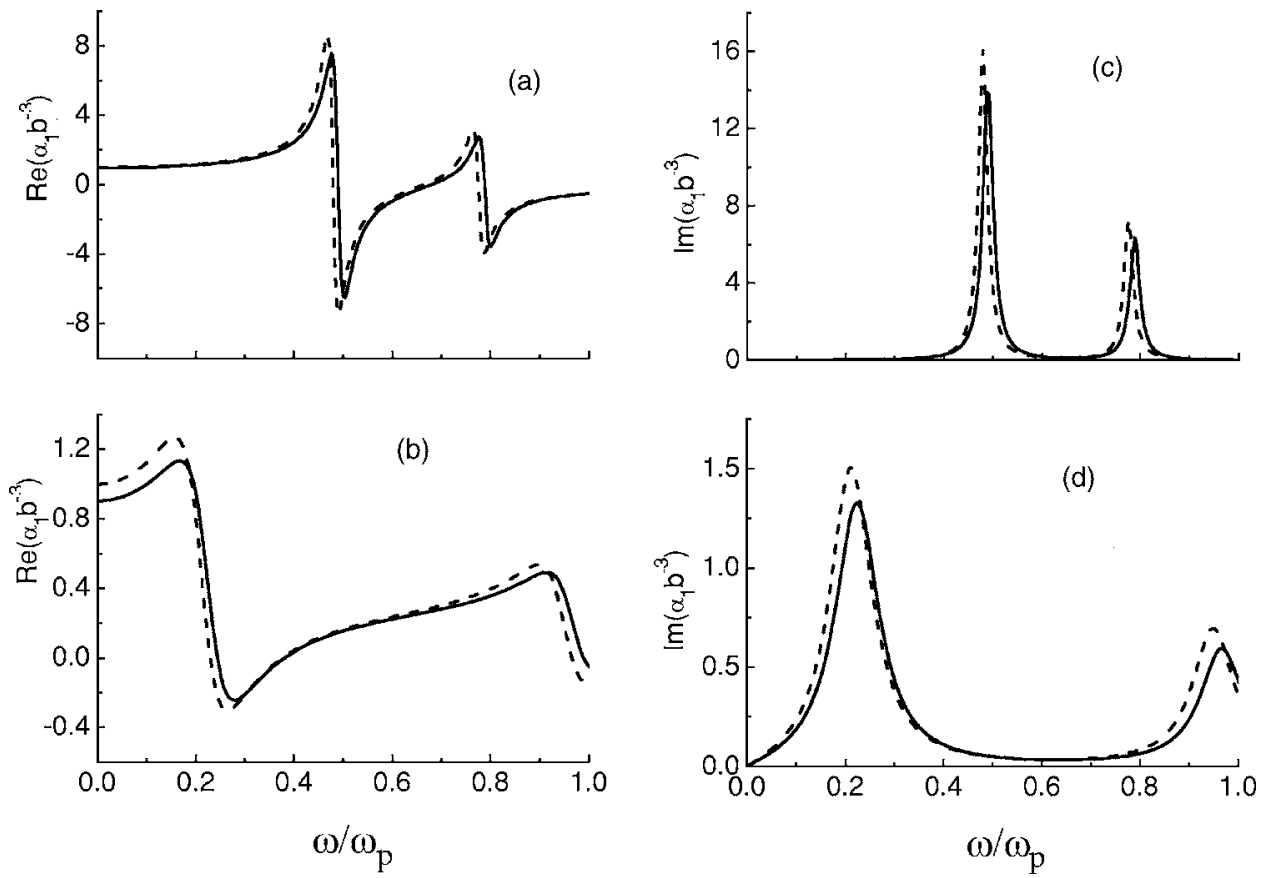

FIG. 2. Real (a) and (b) and imaginary (c) and (d) parts of the dipolar polarizability for a glassfilled silver nanoshell with outer radius $b=10.0 \mathrm{~nm}$ and inner radius: (i) $a=5.0 \mathrm{~nm}$ for (a) and (c); and (ii) $a=9.0 \mathrm{~nm}$ for (b) and (d). Different lines denote results from different models in the same way as in Fig. 1. Note that only the Lindhard-Mermin model is shown for the nonlocal results. the molecule increases towards higher values. In the NL case, however, the antisymmetric modes have resonance frequency too high so that this only occurs when the emission frequency is close to the bulk plasmon frequency. We have also computed (not shown) the results for a larger nanoshell with $a=9 \mathrm{~nm}$ and $b=10 \mathrm{~nm}$, and observed large NL effects as well for this larger shell with very small thickness.

Finally, we would like to study the NL effects on the resonance frequency given in the local theory by Eq. (A3). While (A3) [which includes (A4)] is an idealized result obtained by using the Drude model (without damping) for the metal shell, it has often been used as a guideline in various experimental studies. ${ }^{22}$ In Fig. 4, we have plotted the solutions from Eq. (A3) as a function of $\ell$ (dashed lines). We have also recomputed these resonance frequencies for the case of a realistic Ag-glass shell with damping as used in the computations in Figs. 1-3 by using a numerical search program for the peak positions in the imaginary part of the multipolar polarizability. The results are shown as black dots in Fig. 4, which, surprisingly, compare very close (though not identical) to those given by Eq. (A3). It is interesting to recall that similar results for the surface plasmon resonance frequency of a metal sphere (i.e., being independent of the damping in the Drude model) has been reported previously in the literature. ${ }^{23}$ Moreover, once the NL effects are included, we see that both the antisymmetric and the symmetric modes are blueshifted (as shown by the solid and diamond curves) in their resonance frequencies, as observed

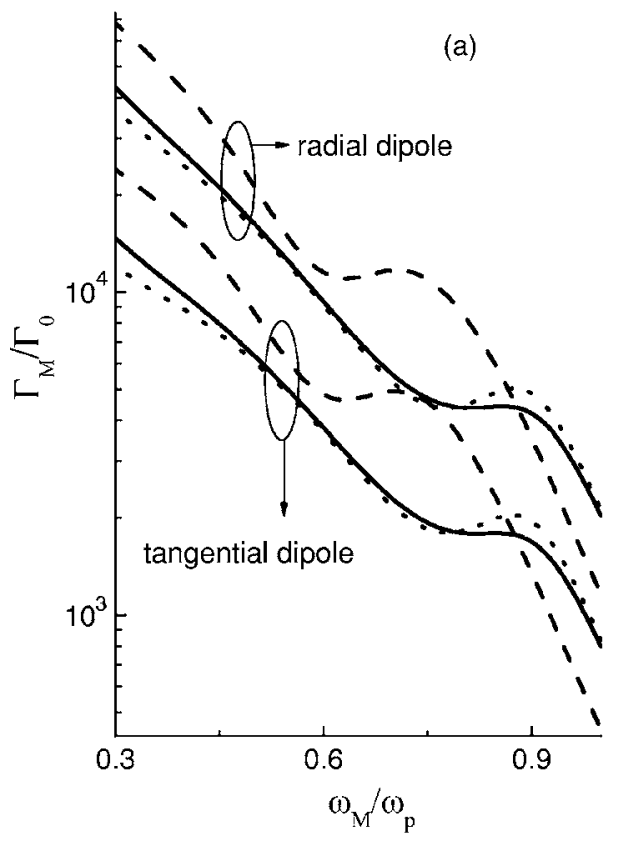

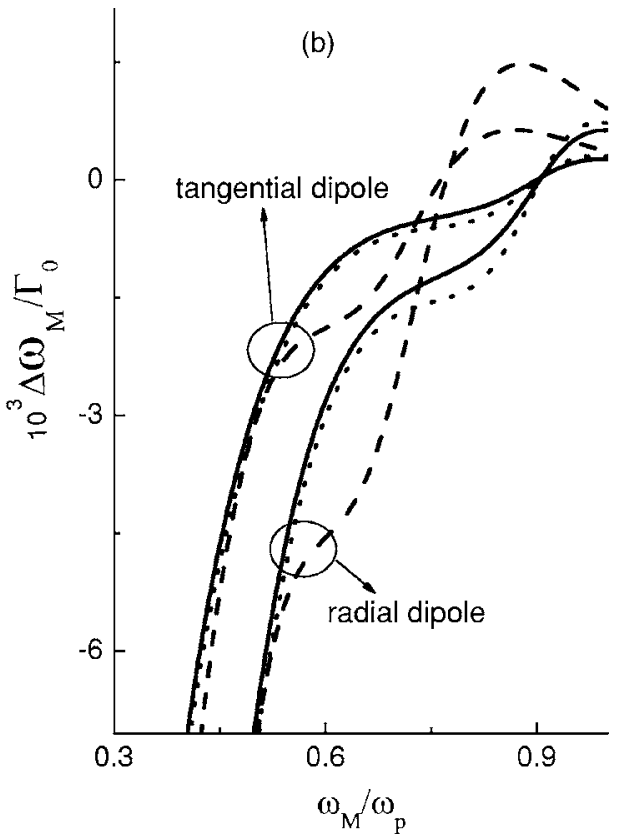

FIG. 3. Normalized decay rate and frequency shift of an emitting molecular dipole at $1 \mathrm{~nm}$ from the same nanoshell for Fig. 1(a), plotted as a function of the normalized emission frequency of the molecule. Different lines denote results from different models in the same way as in Fig. 1. 


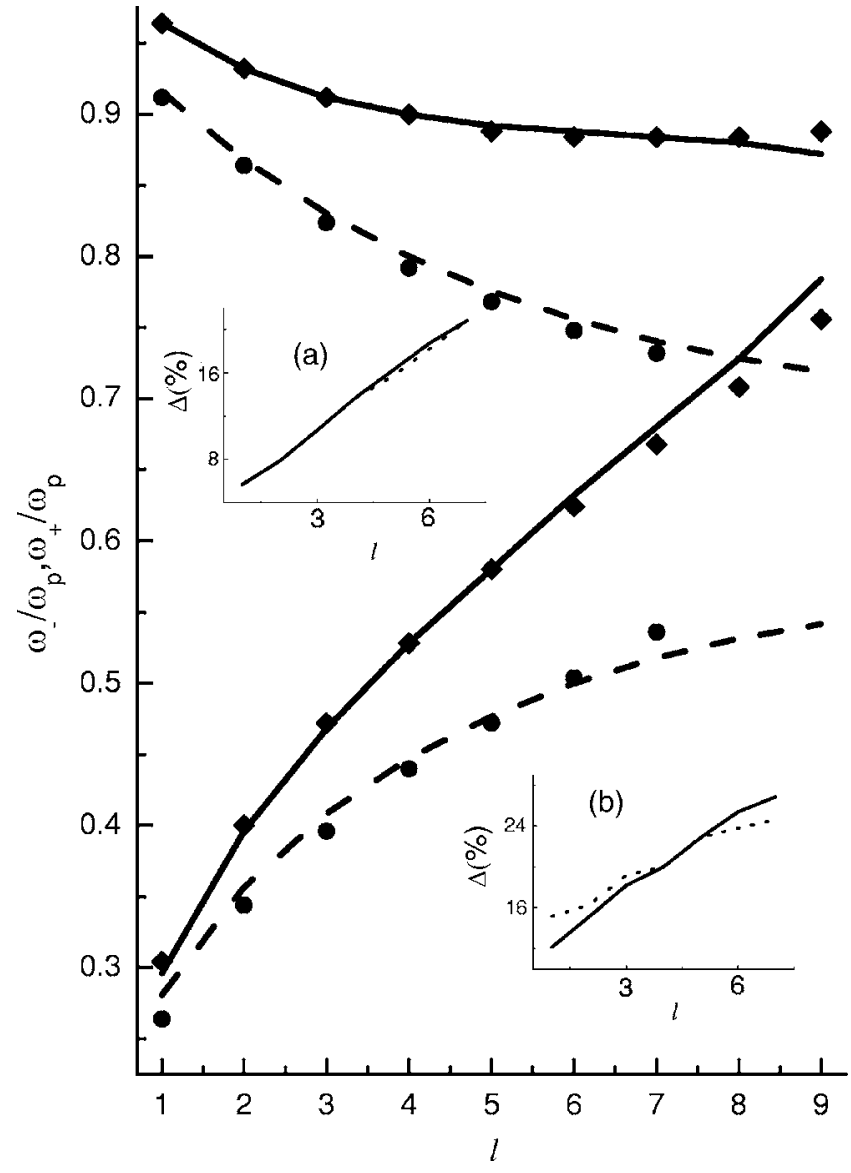

FIG. 4. The two surface plasmon mode frequencies for a silver nanoshell as in Fig. 1(a) evaluated by Drude model (filled circles), hydrodynamic model (diamonds), and Lindhard-Mermin model (solid) as functions of the multipolar order $l$. Also shown (dashed lines) are the results for the ideal Drude case (without damping) as obtained from Eq. (A3). The insets show the percentage deviation $(\Delta)$ from the Drude model in the prediction of the plasmon resonance frequencies by the NL models (solid for LM and dotted for hydrodynamic) as a function of the multipolar order for (a) the antisymmetric and (b) symmetric modes.

previously ${ }^{16}$ and in the above investigations. Again, we see that both the hydrodynamic and LM models predict very close results (within 10\% from each other) for the blueshifted resonance frequencies (especially for the antisymmetric modes). Furthermore, this NL deviation can be very significant for higher multipolar modes, greater than $20 \%$ for $\ell=7$. For the dipole case, however, it is found that the NL effects amount to about 5\% increase for $\omega_{+}$and $12 \%-15 \%$ for $\omega_{-}$, which can be measurable. A detailed analysis of this percentage deviation due to the NL effects is presented in the inset. We should also mention that the present result for the dipole case is in contrast with the one obtained previously via density functional theory (DFT) calculation, in which redshifted frequencies from Eq. (A4) were obtained, ${ }^{24}$ which is likely due to the fact that the "spill-over effect" for the electrons is completely excluded in the SCIB approximation.

\section{CONCLUSION}

In this work, we have applied the previously established NL polarizability for a metallic shell ${ }^{16}$ to study the optical response of these nanoshells. It has been shown that these NL effects can become significant, for both hollow and filled shells, when the size of the shells becomes very small or when the shell thickness becomes very thin. Since current experiments can fabricate these shells with thickness down to about $1 \mathrm{~nm}$, these NL effects can be important in these experiments, which may lead to observation of deviations from the Mie theory even in large (e.g., $\sim 50-100 \mathrm{~nm}$ ) nanoshells. In addition, the NL effect will in general lower the resonant absorption (extinction) efficiency for these nanoshells (up to $\sim 15 \%$ in our model study with $\mathrm{Ag}$ nanoshells). Furthermore, it is seen that in most of our calculations (Figs. 1-3), both the hydrodynamic and the LM models give very similar results (within a few \%), implying that the single particle ( $e-h$ pair) excitation is not significant under the conditions of these calculations when the lower multipolar response from the nanoshell is considered. We have seen the two models start to deviate from each other for multipole $\ell>15$ or even for lower $\ell$ if the damping were small.

We should also remark that our present model is restricted to the long wavelength limit. It is therefore of interest to note that, in a very recent work, $\mathrm{Moroz}^{25}$ has formulated a full electrodynamic theory using a transfer-matrix approach, in which a spherical system of any number of layers can be treated, with the possibility of including NL dielectric response in any of the layers. It would be of interest to apply Moroz's theory to metallic nanoshells as studied in our present work, and compared with the results we obtained using the multipole polarizability. However, since the same additional boundary condition (namely, the continuity of the radial electric field) has been used in Moroz's work, we expect results obtained from the Moroz theory should be similar to ours for such small-size nanoshells.

\section{ACKNOWLEDGMENTS}

This research is partially supported by the National Science Council, Taiwan, under Grant No. NSC 94-2112-M019-002, and Ministry of Economic Affairs, Taiwan, R.O.C. under Grant No. MOEA\# 94-EC-17-A-08-S1-0006 (for RC); and by a research grant from Intel Corporation (for PTL). We also thank Andy Martwick for various help in the processing of the paper.

\section{APPENDIX}

In the local limit Eq. (4) becomes

$$
F(x, y) \rightarrow \frac{1}{\varepsilon} \int_{0}^{\infty} j_{\ell}(k x) j_{\ell}(k y) d k=\frac{\pi}{2 \varepsilon(2 \ell+1)} \frac{x^{\ell}}{y^{\ell+1}},
$$

and one can show from the results in Eqs. (2) and (3) that the polarizability in Eq. (1) indeed reduces back to the familiar one for a local response theory with the factor $\Delta_{\ell}$ given by 


$$
\Delta_{\ell}^{L}=\frac{\left(\varepsilon-\varepsilon^{\prime}\right) \ell[\ell+\varepsilon(\ell+1)] a^{2 \ell+1}+\ell(1-\varepsilon)\left[\varepsilon^{\prime} \ell+\varepsilon(\ell+1)\right] b^{2 \ell+1}}{\left(\varepsilon-\varepsilon^{\prime}\right)(\varepsilon-1) \ell(\ell+1) a^{2 \ell+1}-(\varepsilon \ell+\ell+1)\left[\varepsilon^{\prime} \ell+\varepsilon(\ell+1)\right] b^{2 \ell+1}} .
$$

Note that by using the undamped Drude model for $\varepsilon$, the poles of Eq. (A2) yield the following quadratic equation for the plasmon resonance frequencies of the nanoshell:

$$
\begin{gathered}
{\left[2 \ell^{2}\left(\varepsilon^{\prime}+1\right)+\ell\left(\varepsilon^{\prime}+3\right)+1\right] y^{2}-\left[\left(\varepsilon^{\prime}-1\right) \ell(\ell+1) X^{2 \ell+1}\right.} \\
\left.+\ell^{2}\left(\varepsilon^{\prime}+3\right)+4 \ell+1\right] y-\ell(\ell+1)\left(X^{2 \ell+1}-1\right)=0,
\end{gathered}
$$

where $\sqrt{y}=\left(\frac{\omega_{\ell}}{\omega_{P}}\right)$ is the $\ell$ th-multipole resonance frequency normalized to the bulk plasmon frequency of the metal, and $X \equiv a / b$ is the ratio of the two radii. In the limit for a hollow metallic nanoshell, $\varepsilon^{\prime}=1$ and the solution to (A3) leads back to the well-known results for the plasmon frequencies in the local theory as follows: ${ }^{6}$

$$
\omega_{\ell \pm}^{2}=\frac{\omega_{p}^{2}}{2}\left[1 \pm \frac{1}{2 \ell+1} \sqrt{1+4 \ell(\ell+1) X^{2 \ell+1}}\right] .
$$

The two modes $\omega_{\ell \pm}$ corresponds to the antisymmetric $(+)$ and symmetric $(-)$ coupling between the sphere and cavity modes, respectively, and can be accounted for by a plasmon hybridization model. ${ }^{26}$
${ }^{1}$ S. Oldenburg, R. D. Averitt, S. Westcott, and N. J. Halas, Chem. Phys. Lett. 288, 243 (1998).

${ }^{2}$ S. J. Oldenburg, S. L. Westcott, R. D. Averitt, and N. J. Halas, J. Chem. Phys. 111, 4729 (1999).

${ }^{3}$ S. L. Westcott, J. B. Jackson, C. Radloff, and N. J. Halas, Phys. Rev. B 66, 155431 (2002).

${ }^{4}$ C. Graf and A. v. Blaaderen, Langmuir 18, 524 (2002).

${ }^{5}$ Y. Sun and Y. Xia, Anal. Chem. 74, 5297 (2002).

${ }^{6}$ E. Prodan and P. Nordlander, Chem. Phys. Lett. 352, 140 (2002); E. Prodan, P. Nordlander, and N. J. Halas, ibid. 368, 94 (2003).

${ }^{7}$ R. Baer, D. Neuhauser, and S. Weiss, Nano Lett. 4, 85 (2004).

${ }^{8}$ J. B. Jackson and N. J. Halas, J. Phys. Chem. B 105, 2743 (2001).

${ }^{9}$ E. Hao, S. Li, R. C. Bailey, S. Zou, G. C. Schatz, and J. T. Hupp, J. Phys. Chem. B 108, 1224 (2004).

${ }^{10}$ F. Tam, C. Moran, and N. Halas, J. Phys. Chem. B 108, 17290 (2004).

${ }^{11}$ N. K. Grady, N. J. Halas, and P. Nordlander, Chem. Phys. Lett. 399, 167 (2004).

${ }^{12}$ T. V. Teperik, V. V. Popov, and F. J. Garcia de Abajo, Phys. Rev. B 69, 155402 (2004).

${ }^{13}$ In a series of works, Noguez and coworkers have clarified the limitations of the long-wavelength approximation and the significance of higher order multipoles for various sizes and shapes of the nanoparticles. See, e.g., I. O. Sosa, C. Noguez, and R. G. Barrera, J. Phys. Chem. B 107, 6269 (2003); A. L. Gonzalez, C. Noguez, G. P. Ortiz, and G. Rodriguez-Gattorno, ibid. 109,
17512 (2005); C. Noguez, Opt. Mater. 27, 1204 (2005). See also, K. L. Kelly, E. Coronado, L. L. Zhao, and G. C. Schatz, J. Phys. Chem. B 107, 668 (2003).

${ }^{14}$ W. Ekardt, Phys. Rev. B 34, 526 (1986).

${ }^{15}$ Preliminary results have been reported in the recent SPIE conference (Boston, October 23-27, 2005), see R. Chang and P. T. Leung, in Nanofabrication: Technologies, Devices, and Applications II; edited by W. Y. Lai, L. E. Ocola, and S. Pau, Proc. SPIE series, Vol. 6002 (International Society for Optical Engineering, Bellingham, WA, 2005), p. 171-180.

${ }^{16}$ R. Rojas, F. Claro, and R. Fuchs, Phys. Rev. B 37, 6799 (1988).

${ }^{17}$ Eq. (1) should be equivalent to Eq. 28 of Ref. 16, when the dielectric function of the core in this latter equation is set to be a local response function.

${ }^{18}$ R. R. Chance, A. Prock, and R. Silbey, Adv. Chem. Phys. 37, 1 (1978).

${ }^{19}$ N. D. Mermin, Phys. Rev. B 1, 2362 (1970).

${ }^{20}$ B. B. Dasgupta and R. Fuchs, Phys. Rev. B 24, 554 (1981).

${ }^{21}$ E. Prodan, P. Nordlander, and N. J. Halas, Chem. Phys. Lett. 368, 94 (2003).

${ }^{22}$ Z-j. Jiang and C-y. Liu, J. Phys. Chem. B 107, 12411 (2003).

${ }^{23}$ M. Xu and M. J. Dignam, J. Chem. Phys. 96, 3370 (1992).

${ }^{24}$ E. Prodan and P. Nordlander, Chem. Phys. Lett. 352, 140 (2002).

${ }^{25}$ A. Moroz, Ann. Phys. (N.Y.) 315, 352 (2005).

${ }^{26}$ E. Prodan, C. Radloff, N. J. Halas, and P. Nordlander, Science 302, 419 (2003). 